\title{
Estimating the Effects of Microscopic Stress Concentrations on the Fatigue Endurance of Thin-walled High Strength Steel
}

\author{
Shunsuke TOYODA, ${ }^{1)}$ Yoshikazu KAWABATA, ${ }^{1)}$ Kei SAKATA, ${ }^{2)}$ Akio SATO, ${ }^{3)}$ Naotake YOSHIHARA ${ }^{4)}$ and \\ Jun'ichi SAKAI ${ }^{5}$ \\ 1) Steel Research Laboratory, JFE Steel Corporation, Kawasaki-cho 1-1, Handa, Aichi 475-8611 Japan. \\ 2) Formerly JFE Steel Corporation, now at JFE Techno-Research Corporation, 1, Kawasaki-cho, Chuo-ku, Chiba, Chiba 260- \\ 0835 Japan. \\ 3) Chita Works, JFE Steel Corporation, Kawasaki-cho 1-1, Handa, Aichi 475-8611 Japan. \\ 4) JFE Techno-Research Corporation, Minamiwatarida-cho 1-1, Kawasaki-ku, Kawasaki, Kanagawa 210-0855 Japan. \\ 5) Faculty of Science and Engineering, Waseda University, Okubo 3-4-1, Shinjuku-ku, Tokyo 169-8555 Japan.
}

(Received on April 24, 2009; accepted on July 6, 2009)

\begin{abstract}
The effects of microscopic surface stress concentrations on the fatigue endurance of thin-walled high strength steel were systematically estimated by numerical analysis and plane-bending fatigue tests with Schenck-type specimens, using the commercially available static implicit method FEA software I-DEAS ver. 11 for the stress distribution calculations. The microscopic stress concentration factor $\alpha_{i}$ from notch depth $t=50 \mu \mathrm{m}$ and notch root radius $\rho=6 \mu \mathrm{m}$ microscopic surface ridges, monotonously increased with increases in the roughness ridge direction, $\theta$, from 1 to 7 in the bending mode. A fitted curve was developed for deriving the calculated stress concentration, $\alpha_{\theta}$, from the superposition of the principal stresses. In the twisting mode, $\alpha_{i}$ varied from about 4 to 7 . The $\theta$ dependency of $\alpha_{i}$ was smaller than that in bending mode. The empirical rule that the specimen collection direction has a lesser effect in twisting mode fatigue was supported by the $\alpha_{i}$ value. It is reported that the fatigue notch factor $\beta$ increased linearly with increases in the macroscopic stress concentration factor $\alpha_{a}$ of up to 3 . On the other hand, $\beta$ slowly increased with increases in $\alpha_{i}$ until it exceeded about 2. This marked difference might be due to differences between their respective stress gradients, which was well described by Nisitani and Endo by using a parameter $\rho$. A plane-bending fatigue test was performed with an artificial surface micro-groove of $\theta=0,90^{\circ}$ using $590 \mathrm{MPa}$ class strength circumferentially flattened electric resistance welded tube. The $\theta=0^{\circ}$ micro-groove had little effect on the fatigue endurance in bending. On the other hand, the fatigue cracks of all the $\theta=90^{\circ}$ specimens initiated at the basilar part of the micro-groove without any nonpropagating cracks. The fatigue notch factor $\beta$ seems to be determined by only $\alpha_{i}$ independent of $\rho$ in the microscopic stress concentration field.
\end{abstract}

KEY WORDS: microscopic stress concentration; fatigue; steel; surface roughness.

\section{Introduction}

The surface roughness is one of the main influencing factors on the fatigue endurance of thin-walled automotive structural parts. ${ }^{1)}$ The effects of the surface roughness parameter and the surface groove depth on fatigue endurance have been intensively investigated. ${ }^{1-9)}$ It is well known that the increase in surface roughness deteriorate fatigue endurance. It is also known that this sensitivity to roughness increases with increments of the material strength. Kinefuchi ${ }^{9}$ clarified the effects of the maximum surface roughness on the fatigue endurance of $780 \mathrm{MPa}$ class strength steel. Some studies have performed to describe the effects of surface roughness in terms of microscopic stress concentrations. ${ }^{10-12)}$ Arola and Williams ${ }^{10)}$ experimentally investigated the effects of the microscopic stress concentration factor, $\alpha_{i}$ on the fatigue endurance of AISI $4130 \mathrm{CR}$ steel with machined surface by converting the surface roughness profile to effective stress concentration factors.
At the same time, the effects of macroscopic stress concentration on fatigue endurance have been systematically examined and quantitatively discussed. ${ }^{13-22)}$ Frost and Dugdale ${ }^{13)}$ systematically investigated the effects of the nominal stress, $\sigma_{\text {nominal }}$, and the macroscopic stress concentration factor, $\alpha_{a}$, on the fatigue endurance and the generation of nonpropagating cracks. They found that there are three cases depending on the combination of $\sigma_{\text {nominal }}$ and $\alpha_{a}$ : (1) fractured, (2) not fractured with nonpropagating cracks, and (3) not fractured without nonpropagating cracks. However, the effects of the microscopic stress concentration factor $\alpha_{i}$, which indicates micrometer-size stress concentrations, have not been fully clarified yet.

In this study, an artificial microscopic stress concentration was postulated, and the $\alpha_{i}$ of the microscopic stress concentration was calculated by finite element analysis. Stress loading modes, i.e., bending and twisting, and the direction and the length of the microscopic surface ridge were varied as the influencing factors in the analysis. The effects 


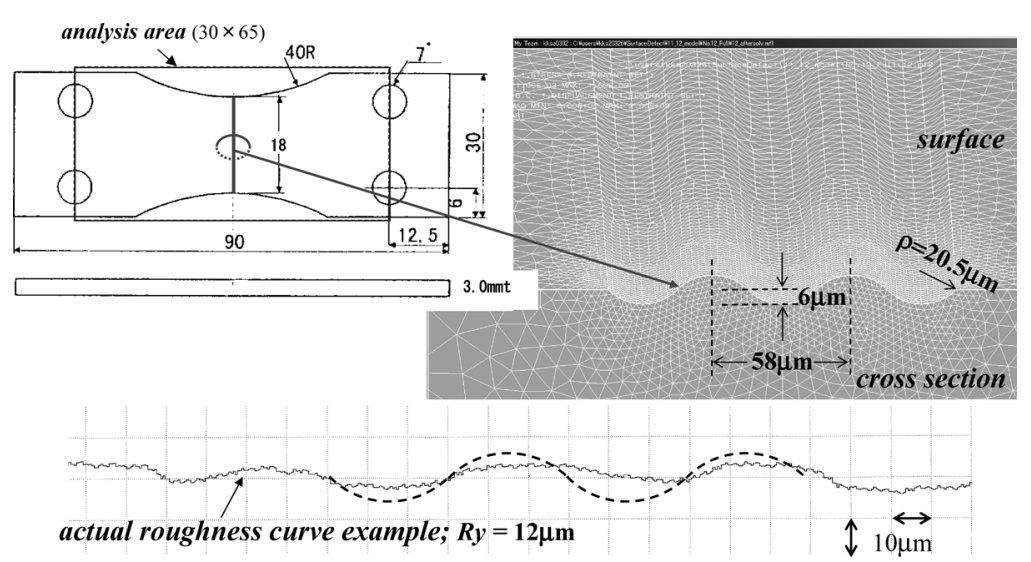

Fig. 1. Configuration of the Schenck-type plane-bending fatigue test specimens for FEA with concave-convex surfaces.

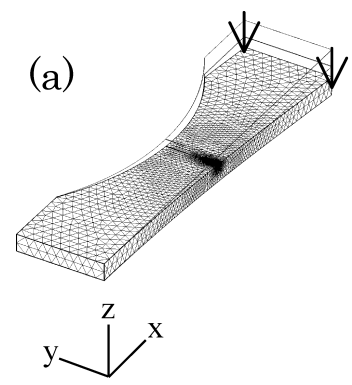

Fig. 2. Deformed specimen. (a) Bending-mode, (b) twistingmode.

of the calculated $\alpha_{i}$ were combined with the reported and examined fatigue data in order to estimate the effects of the stress gradient near the macro- and microscopic stress concentrations on the fatigue strength. The effects of the ridge direction and $\alpha_{i}$ were experimentally examined in addition to being subject to numerical analysis.

\section{Microscopic Stress Concentration Analysis}

A finite element analysis was performed to estimate the microscopic stress concentration factor, $\alpha_{i}$, of the concave-convex surface simulating the surface roughness on a Schenck-type plane-bending fatigue test specimen, as shown in Fig. 1. The concave-convex shape was a two-dimensional continuous quarter-circle profile with a $6 \mu \mathrm{m}$ amplitude $(t), 20.5 \mu \mathrm{m}$ radius of curvature $(\rho)$, and a $58 \mu \mathrm{m}$ cycle length. Bending and twisting modes were adopted as the external forces, as shown in Fig. 2. The forced displacement was $1 \mathrm{~mm}$. Typical high-strength low-carbon steel properties (Young's modulus $E=206 \mathrm{GPa}$ and Poisson's ratio $v=0.3$ ) were used for the calculation. The commercially available static implicit method FEA software, IDEAS ver. 11, was used to calculate the stress distribution with a tetrahedral mesh. The amplitude of the surface concavity and convexity was small, corresponding to only $0.4 \%$ of the half-specimen thickness.

To evaluate the effect of the roughness ridge direction, further calculations were carried out. The angle of deflection between the longitudinal direction of the specimen and the roughness ridge direction $\theta$ was defined as shown in Fig. 3. A higher microscopic stress concentration was adopted to clearly show the effect of the angle of deflection

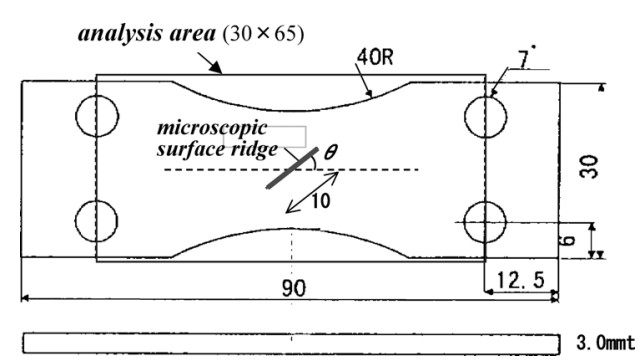

Fig. 3. Direction of microscopic surface ridges on the fatigue test specimens.

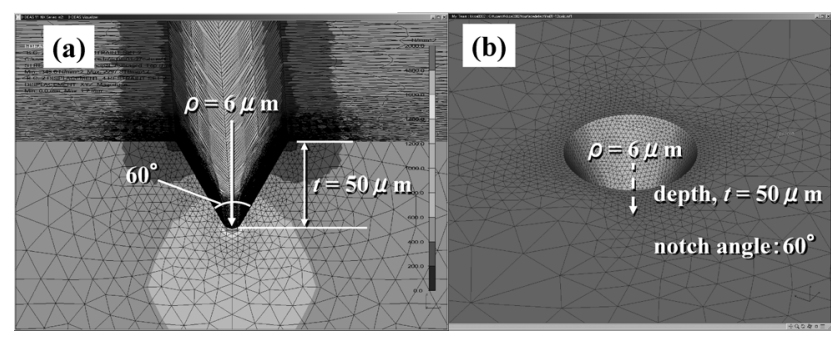

Fig. 4. Artificial microscopic stress concentrations for estimating the effect of (a) angle deflection and (b) roughness ridge length.

with notch depth, $t=50 \mu \mathrm{m}$ and notch root radius, $\rho=6 \mu \mathrm{m}$, as shown in Fig. 4(a). Furthermore, to figure out the effect of the roughness ridge length, the $\alpha_{i}$ of the conical concavity shape shown in Fig. 4(b) was also calculated.

A plane-bending fatigue test was performed on a Schenck-type specimen with an artificial surface microgroove of $\theta=0,90^{\circ}$. $590 \mathrm{MPa}$ class strength $2.6 \mathrm{~mm}$ thickness electric resistance welded steel tube was used for the experiment. The $0.2 \%$ proof stress, $\sigma_{0.2}=570 \mathrm{MPa}$, the tensile strength, $\Delta_{B}=631 \mathrm{MPa}$, and the elongation, $\mathrm{El}=23 \%$, respectively. The ferrite grain diameter, $d_{f}=3 \mu \mathrm{m}$, and the maximum height of the surface roughness, $R_{y}=8 \mu \mathrm{m}$. The Schenck-type plane-bending specimen was circumferentially flattened from the tube and was grooved on the outer surface with a cutter knife. The groove length was $10 \mathrm{~mm}$. In case of $\theta=0^{\circ}$, three parallel grooves were cut on the specimen at $5 \mathrm{~mm}$ intervals. The $\alpha_{i}$ of the groove was varied from 5.1 to 8.1 corresponding to the change of the cutting load from 200 to $1400 \mathrm{gf}$. The notch depth $t$ and notch root radius $\rho$ were varied from 25 to $100 \mu \mathrm{m}$ and from 6 to $8 \mu \mathrm{m}$, respectively. The $\alpha_{i}$ value of these specimens was 


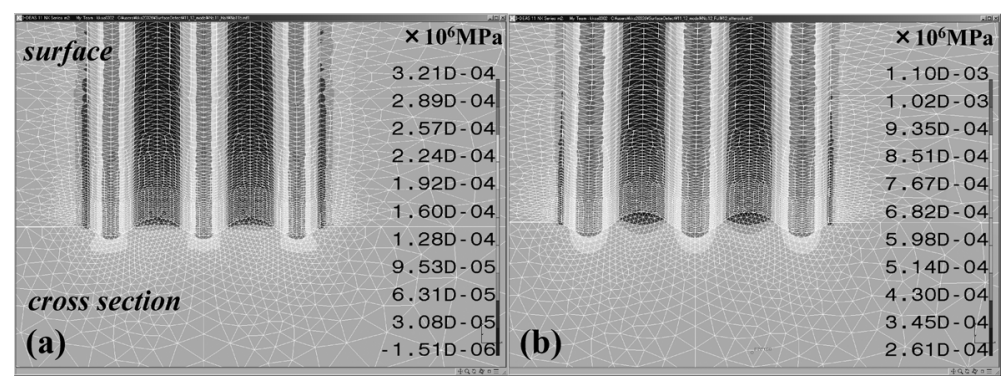

Fig. 5. Major principal stress distribution for the concave-convex shape with $t=6 \mu \mathrm{m}$ and $\rho=20.5 \mu \mathrm{m}$.
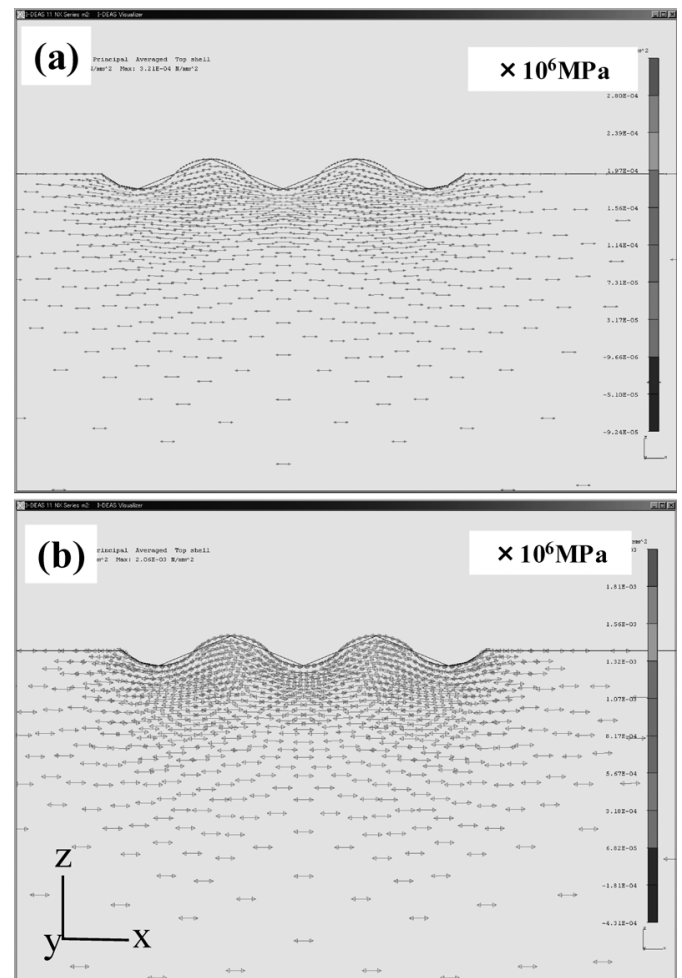

Fig. 6. Major principal stress distribution for the direction within the $x-z$ cross section. (a) Bending-mode, (b) twistingmode.

calculated by Newber's equation described below. From a practical viewpoint the plane-bending fatigue test was performed on the grooved specimen without any stress relief annealing. The influences of work hardening and residual stress were corrected in the following discussion.

\section{Analysis and Results}

\subsection{Numerical Analysis of the Microscopic Stress Concentration Corresponding to the Surface Roughness}

The major principal stress distributions of the concaveconvex shape with $t=6 \mu \mathrm{m}$ and $\rho=20.5 \mu \mathrm{m}$ are shown in Fig. 5. A local stress concentration is observed at the basilar part of the concavities. Figure 6 shows the major principal stress distribution in the $x-z$ cross section. The alterations of the stress amplitude and direction caused by the surface ridge diminish severalfold with the ridge depth from the surface. The maximum principal stress without the surface ridge $\left(\sigma_{\text {nominal }}\right)$ was separately calculated, and then $\alpha_{i}$ was obtained. Table 1 shows the $\alpha_{i}$ calculated by the
Table 1. $\alpha_{i}$ values of the concave-convex shape with $t=6 \mu \mathrm{m}$ and $\rho=20.5 \mu \mathrm{m}$ calculated by FEA.

\begin{tabular}{c|c|c}
\hline & Bending & Torsional \\
\hline FEA analysis & $2.15 / 1.86$ & $1.56 / 1.54$ \\
\hline Neuber's equation & $2.08 /-$ & $1.54 /-$ \\
\hline \multicolumn{2}{|c}{ Major principal stress / Mises stress }
\end{tabular}

FEA in this study along with the $\alpha_{i}$ obtained from Neuber's approximate equation $^{14)}$ for a shallow semi-elliptical surface notch in a half-infinite body as follows:

$$
\alpha=1+C \sqrt{(t / \rho)}
$$

where $C=2$ in bending mode and $C=1$ in twisting mode.

\subsection{Effect of the Direction of the Microscopic Surface Ridge}

The principal stress distributions for $\theta=0,45$, and $90^{\circ}$ in bending mode are shown in Fig. 7. The stress gradient is very sharp when $\theta=90^{\circ}$; however, it is gradual when $\theta=0^{\circ}$. Figure 8 shows the principal and shear stress distributions along the thickness direction when $\theta=90^{\circ}$. The effect of the surface concavity increases near the surface. Incidentally, the reason why the sign of the principal stress in Figs. 7 and 8 when $\theta=90^{\circ}$ is negative is that the bending direction of this case is opposite that compared to the other cases.

Figure 9 shows the effect of $\theta$ on $\alpha_{i}$ at the basilar part of the concavities in bending mode. $\alpha_{i}$ monotonously increases with the increments of $\theta$. The fitted curve expresses the calculated stress concentration, $\alpha_{\theta}$, derived from the superposition of the principal stresses as shown in Fig. 10.

In general, the conversion of the stress tensor from the $x-y$ coordinate system to the $x^{\prime}-y^{\prime}$ coordinate system on a two-dimensional surface is expressed as follows:

$$
\left(\begin{array}{cc}
\sigma_{x}^{\prime} & \tau_{x y}^{\prime} \\
\tau_{x y}^{\prime} & \sigma_{y}^{\prime}
\end{array}\right)=\left(\begin{array}{cc}
\cos \theta & \sin \theta \\
-\sin \theta & \cos \theta
\end{array}\right)\left(\begin{array}{cc}
\sigma_{x} & \tau_{x y} \\
\tau_{x y} & \sigma_{y}
\end{array}\right)\left(\begin{array}{cc}
\cos \theta & -\sin \theta \\
\sin \theta & \cos \theta
\end{array}\right)
$$

In the case of the unidirectional stress state, it is expressed as follows:

$$
\left(\begin{array}{ll}
\sigma_{x} & \tau_{x y} \\
\tau_{x y} & \sigma_{y}
\end{array}\right)=\left(\begin{array}{cc}
\sigma_{0} & 0 \\
0 & 0
\end{array}\right)
$$

where $\sigma_{0}$ is the maximum principal stress in the longitudinal direction of the specimen caused by the bending mo- 


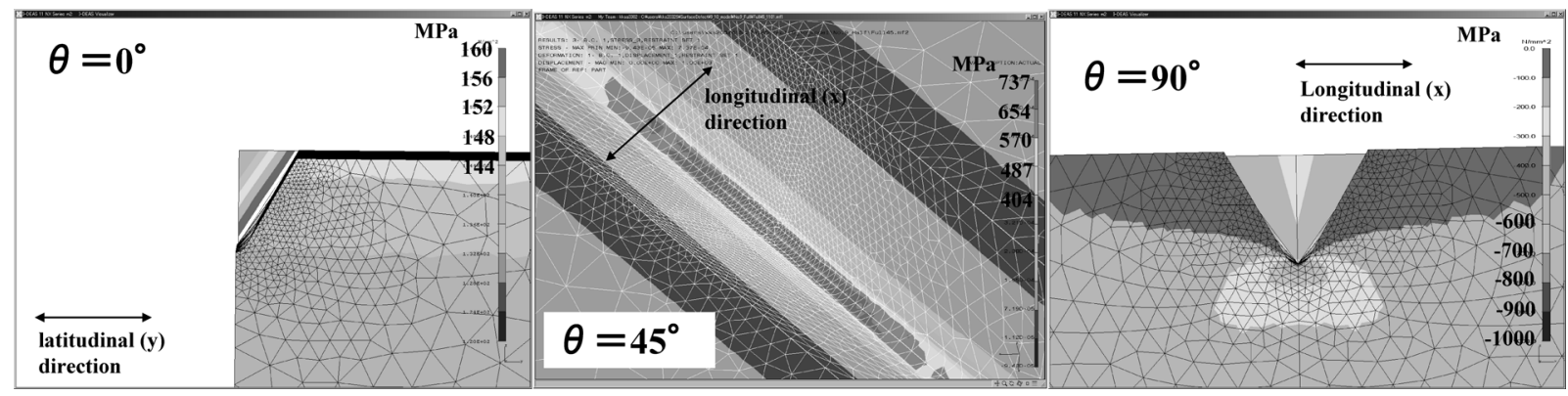

Fig. 7. Principal stress distribution in the vicinity of the microscopic surface ridges in bending mode.

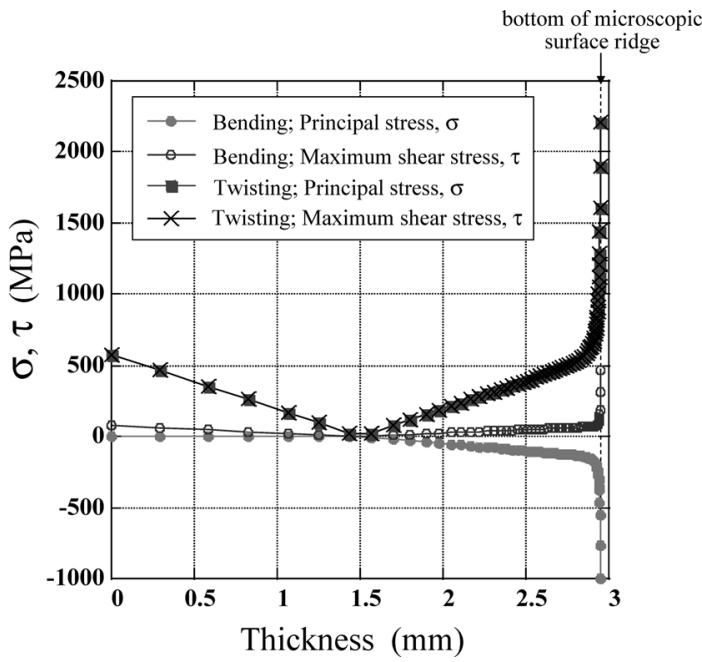

Fig. 8. Principal and shear stress distributions along the thickness direction when $\theta=90^{\circ}$.

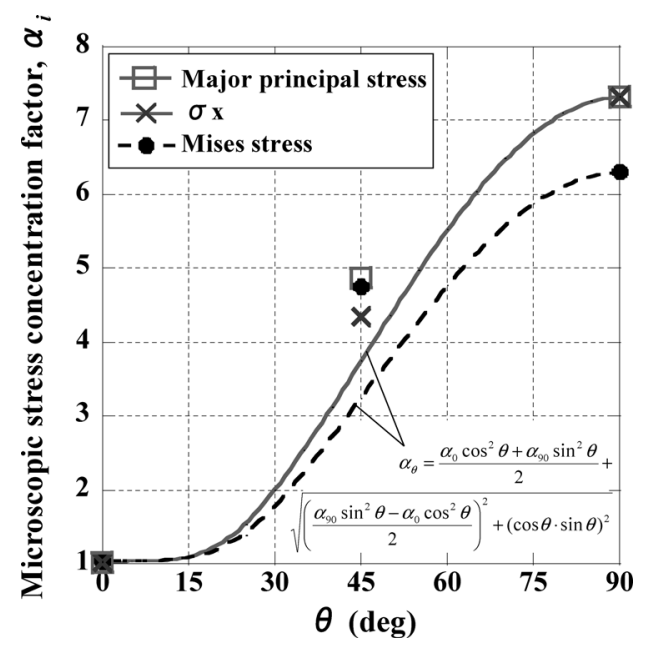

Fig. 9. Effect of $\theta$ on $\alpha_{i}$ at the basilar part of the concavities in bending mode.

ment. The converted stress tensor is expressed by the following equation:

$$
\left(\begin{array}{ll}
\sigma_{x}^{\prime} & \tau_{x y}^{\prime} \\
\tau_{x y}^{\prime} & \sigma_{y}^{\prime}
\end{array}\right)=\sigma_{0}\left(\begin{array}{cc}
\cos ^{2} \theta & -\cos \theta \sin \theta \\
-\cos \theta \sin \theta & \sin ^{2} \theta
\end{array}\right)
$$

When the surface concave direction is $\theta$, the converted stress tensor could be represented by

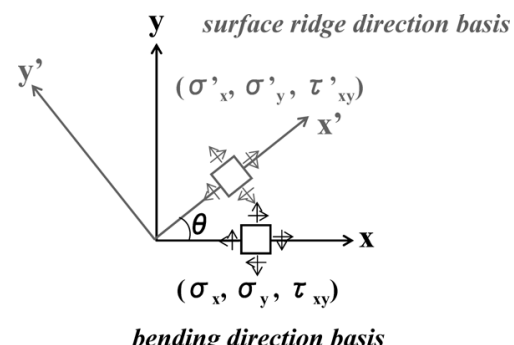

Fig. 10. Conversion of the stress tensor from the $x-y$ coordinate system to the $x^{\prime}-y^{\prime}$ coordinate system on a two-dimensional surface.

$$
\left(\begin{array}{ll}
\sigma_{x}^{\prime} & \tau_{x y}^{\prime} \\
\tau_{x y}^{\prime} & \sigma_{y}^{\prime}
\end{array}\right)=\sigma_{0}\left(\begin{array}{cc}
\alpha_{0} \cos ^{2} \theta & -\cos \theta \sin \theta \\
-\cos \theta \sin \theta & \alpha_{90} \sin ^{2} \theta
\end{array}\right)
$$

where $\alpha_{0}$ is the stress concentration factor of the $\theta$ direction and $\alpha_{90}$ is that of the $\theta+90^{\circ}$ direction.

The terms $\alpha_{0} \cos ^{2} \theta$ and $\alpha_{90} \sin ^{2} \theta$ express the stress components both parallel and perpendicular to the concave direction. The maximum principal stress, $\sigma_{\theta}$, of the stress tensor changed by the concave stress concentration can be obtained by

$$
\begin{aligned}
\sigma_{\theta}=\sigma_{0} & {\left[\frac{\alpha_{0} \cos ^{2} \theta+\alpha_{90} \sin ^{2} \theta}{2}\right.} \\
& \left.+\sqrt{\left(\frac{\alpha_{90} \sin ^{2} \theta-\alpha_{0} \cos ^{2} \theta}{2}\right)^{2}+(\cos \theta \cdot \sin \theta)^{2}}\right]
\end{aligned}
$$

Accordingly, the stress concentration factor, $\alpha_{\theta}$, of the maximum principal stress is as follows:

$$
\begin{aligned}
\alpha_{\theta}= & \frac{\sigma_{\theta}}{\sigma_{0}}=\frac{\alpha_{0} \cos ^{2} \theta+\alpha_{90} \sin ^{2} \theta}{2} \\
& +\sqrt{\left(\frac{\alpha_{90} \sin ^{2} \theta-\alpha_{0} \cos ^{2} \theta}{2}\right)^{2}+(\cos \theta \cdot \sin \theta)^{2}}
\end{aligned}
$$

The deviation between the $\alpha_{\theta}$ value obtained by FEA and that calculated by Eq. (7) may be due to the angle deflection between the maximum principal stress and the $\sigma_{y}^{\prime}$ direction in Eq. (5). The $\alpha_{\theta}$ value obtained by FEA may be added to the $\tau_{x y}^{\prime}$ component of the stress concentration factor.

Figure 11 shows the effect of $\theta$ on $\alpha_{i}$ at the basilar part 


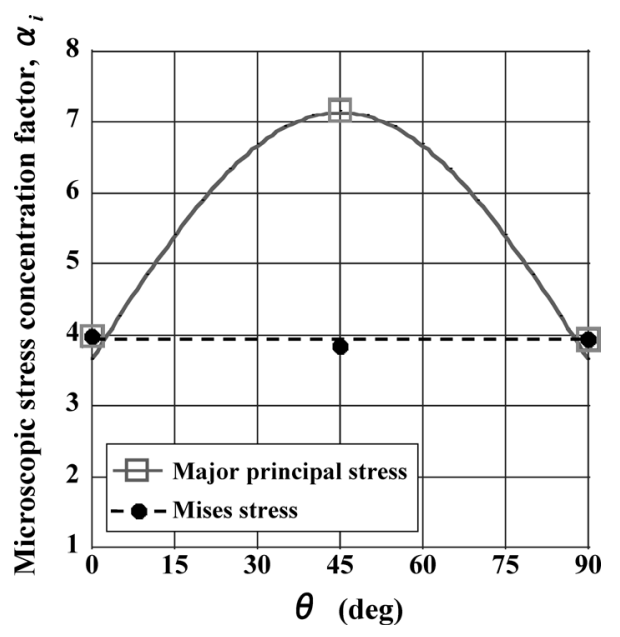

Fig. 11. Effect of $\theta$ on $\alpha_{i}$ at the basilar part of the concavities in twisting mode.

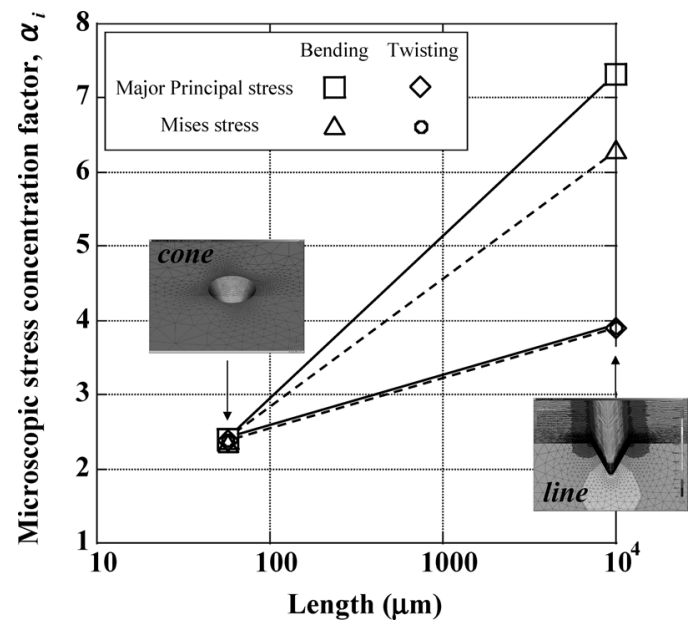

Fig. 12. Re-plotted data from Table 3 as a function of concavity length.

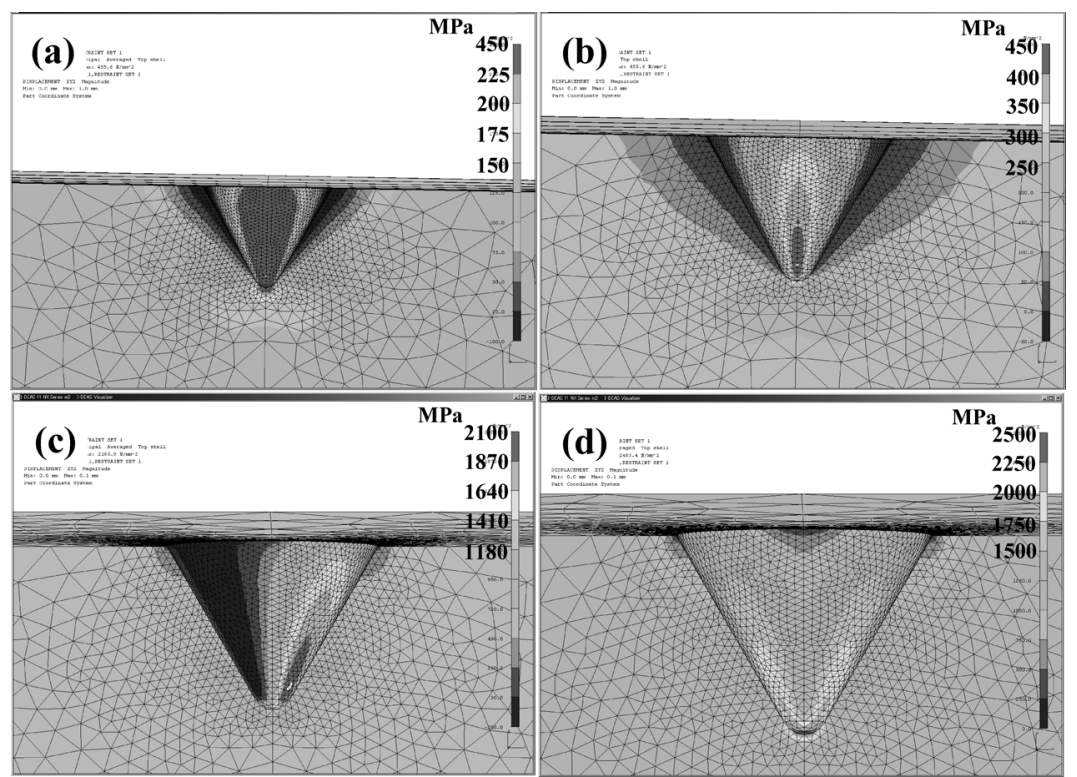

Fig. 13. Stress distributions in the conical concave shape of the longitudinal $(x-z)$ cross section. (a) Major principal stress in bending, (b) $\sigma_{x}$ in bending, (c) major principal stress in twisting, (d) Mises stress in twisting.

of the concavities in twisting mode. The $\alpha_{i}$ value of the maximum principal stress varies from about 4 to 7 . The angle dependency is similar to that in bending mode, where it is out of phase by 45 degrees. This shows that the principal stress directions in the bending and twisting modes are different from each other by 45 degrees. The $\alpha_{i}$ value of the Mises stress is barely dependent on $\theta$.

The $\theta$ dependency of $\alpha_{i}$ in twisting mode is smaller than that in bending mode. The empirical rule that the specimen collection direction has a lesser effect in twisting mode fatigue $^{3)}$ is confirmed by the obtained $\alpha_{i}$ value.

In reality, when the stress exceeds specimen's proof stress by a stress concentration, plastic deformation and stress relaxation may occur. So, the increment of $\alpha_{i}$ works as the increment of plastic strain and the increment of deformed area at the notch root.

\subsection{Effect of the Length of the Microscopic Surface Ridge}

The $\alpha_{i}$ obtained by FEA for the conical concavity shape is shown in Fig. 12, with the linear concavities as a function of concavity length. All the $\alpha_{i}$ values decrease with decreases in the ridge length. Figure 13 shows the stress distribution in the conical concavity shape. The maximum stress position is altered with the principal stress or the Mises stress in twisting mode.

Figure 14 shows the ratio of the Mises stress $\sigma_{v}$ and the maximum principal stress $\sigma_{1}$ in the bending and twisting modes, for the samples with ridges and with smooth surfaces, as a function of $\theta$. In the bending mode, the ratio of $\sigma_{v} / \sigma_{1}$ is approximately 1 and is independent of $\theta$, which corresponds to a uniaxial stretching mode under a plane stress condition as follows:

$$
\sigma_{1}^{2}+\sigma_{3}^{2}-\sigma_{1} \sigma_{3}=\sigma_{v}^{2}
$$

when $\sigma_{2}=0$. If $\sigma_{v} / \sigma_{1}=1$ is assigned to Eq. (8), then $\sigma_{3}=0$.

In the twisting mode, the ratio of $\sigma_{v} / \sigma_{1}$ is about $\sqrt{3}$, except for where $\theta=45^{\circ}$ in the ridge case, which corresponds to a pure shear mode under a plane stress condition. If 


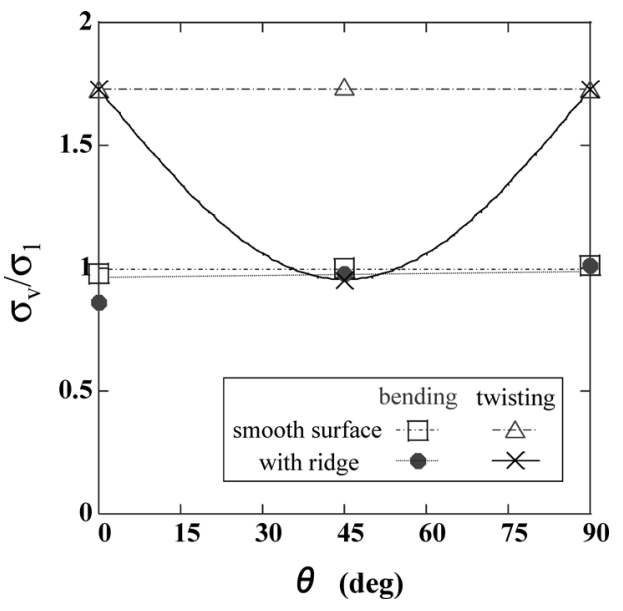

Fig. 14. Ratio of the Mises stress $\sigma_{v}$ and the maximum principal stress $\sigma_{1}$ in bending and twisting modes.

$\sigma_{v} / \sigma_{1}=\sqrt{3}$ is assigned to Eq. (8), then $\sigma_{3}=-\sigma_{1}$. In the case of the conical concavity shape, $\sigma_{v} / \sigma_{1}$ is about 1 in bending mode and is about $\sqrt{3}$ in twisting mode.

\section{Discussion}

\subsection{Effects of the Stress Concentration Factor and the Stress Gradient on Fatigue Endurance}

The effect of the macroscopic stress concentration factor $\alpha_{a}$ on the fatigue endurance of high tensile steel has been intensively investigated. Ishibashi ${ }^{16)}$ studied the relationship between $\alpha_{a}$ and the fatigue notch factor $\beta$ (=fatigue strength of smooth specimen/fatigue strength of notched specimen) in a rotating bending fatigue test of $780 \mathrm{MPa}$ class strength steel. It was revealed that $\beta$ linearly increases with increments of $\alpha_{a}$ up to around 3. Kinefuchi and Tamura ${ }^{20)}$ also investigated the relationship in a tensile-compression mode on $590 \mathrm{MPa}$ class strength steel sheet and showed that $\beta$ is almost the same as $\alpha_{a}$ up to about 3 .

In this study, the relationship between the microscopic stress concentration factor, $\alpha_{i}$, and $\beta$ was examined. Figure 15 shows the extracted and rearranged data by Tomita et $a l{ }^{1)}$ for the relationship between the fatigue strength, $\Delta \sigma$, and the maximum height of the surface roughness, $R_{y}$, of $780 \mathrm{MPa}$ class strength steel sheets. $\alpha_{i}$ is calculated by Neuber's equation, Eq. (1), with the assumption that $t=0.5 R_{y}$ and $\rho=20.5 \mu \mathrm{m}$ (same as Fig. 2). The data at $\alpha_{i}=1$ in this study were obtained with a smoothly surface-ground specimen. The fatigue strength gradually decreased with increases of $\alpha_{i}$.

The relationship between $\alpha_{i}$ and $\beta$ is replotted from Fig. 15 and is shown in Fig. 16. According to the previous reports, ${ }^{16,20)} \beta$ increases linearly with increases of $\alpha_{a}$. Meanwhile, $\beta$ slowly increases with increases of $\alpha_{i}$ until it reaches around 2. Figure 17 shows the stress distributions in the vicinity of the basilar part of the microscopic and macroscopic concavities when both have the same stress concentration factor of 2.15 (shown in Fig. 5(a)). The stress concentration sharply decreases in the case of the microscopic stress concentration. However, that decrease is smaller in the case of the macroscopic stress concentration of the same scale. Figure 17 also shows a schematic illustration of the ferrite grain ${ }^{23)}$ of high tensile strength steel. $\alpha_{i}$

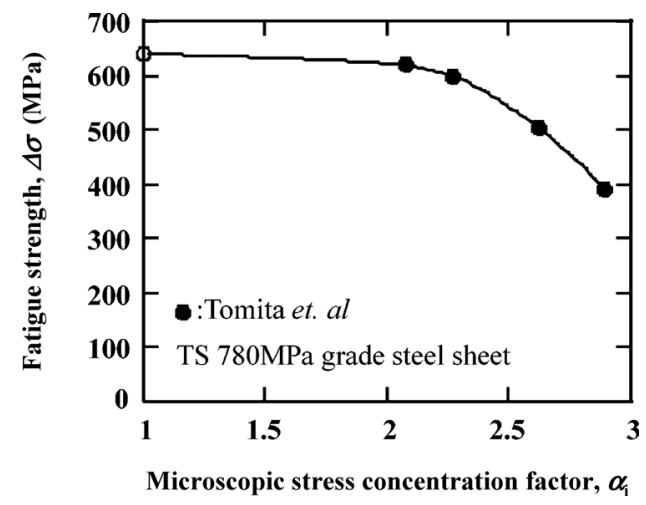

Fig. 15. Rearranged data by Tomita et al. for the relationship between the fatigue strength, $\Delta \sigma$, and the maximum height of the surface roughness, $R_{y}$, of TS $780 \mathrm{MPa}$ grade steel sheets.

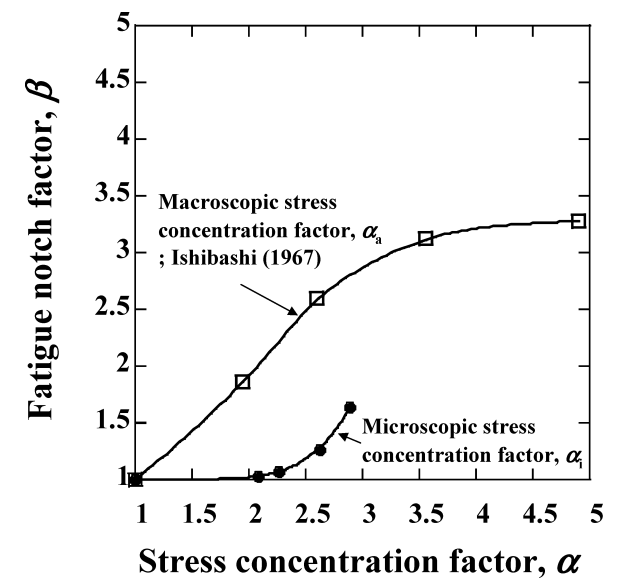

Fig. 16. Relationship between $\alpha_{i}$ and $\beta$ replotted from Fig. 15.

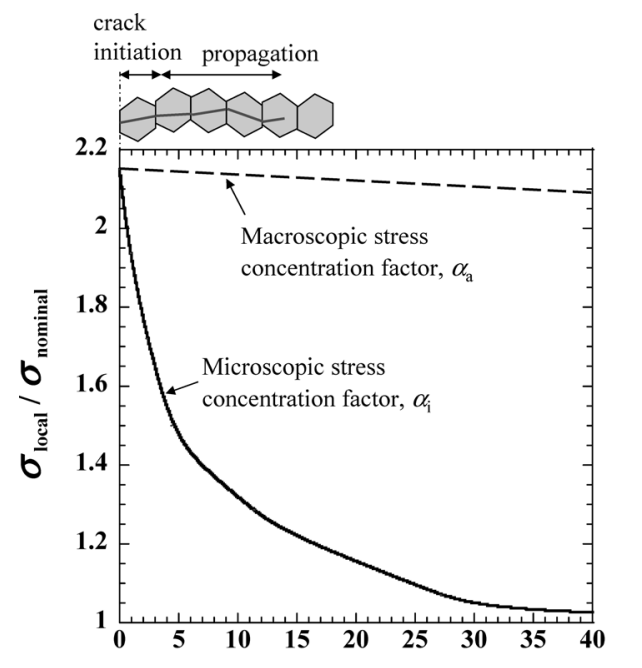

Distance from basilar part of the concave $(\mu \mathrm{m})$

Fig. 17. Stress distribution in the vicinity of the basilar part of the microscopic and macroscopic concavities.

decreases by half within a couple of grain size distances, which is considered to correspond to the initiated fatigue crack size, in the case of the microscopic stress concentration. This marked difference of the stress gradient might have large effects on the fatigue crack initiation and also on its propagation. Consequently, different effects on $\beta$ by both $\alpha_{a}$ and $\alpha_{i}$ are brought about. 


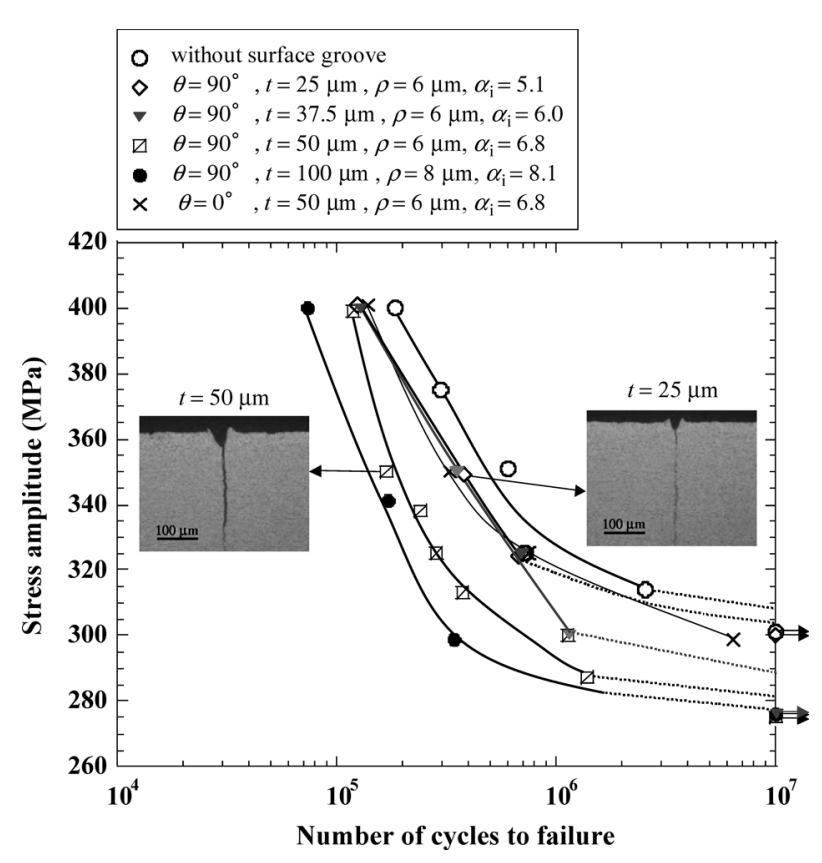

Fig. 18. Fatigue endurance $(\mathrm{S}-\mathrm{N})$ curves of Schenck-type specimens with artificial surface micro-grooves.

These effects of stress gradient on fatigue endurance was well described by Nisitani and Endo by using a parameter $\rho .^{24)}$ The deviation of macroscopic- and microscopic concentration curve in Fig. 16 can be unified by the relation reported by Nisitani. For example, the microscopic to macroscopic fatigue endurance ratio at $\alpha=2.6$ is 2.0 according to Fig. 16. On the other hand, the ratio extracted from the Nisitani's relation based on the $\rho$ value was 1.9 . They are coincident with each other very well.

\subsection{Effect of the Microscopic Stress Concentration on Fatigue Endurance}

Figure 18 shows the fatigue endurance $(\mathrm{S}-\mathrm{N})$ curves of the Schenck-type specimens with artificial surface microgrooves. All the fatigue cracks of $\theta=90^{\circ}$ specimens initiated at the basilar part of the micro-grooves. Including runout specimens, nonpropagating cracks were not observed. On the other hand, the fatigue cracks of all the $\theta=0^{\circ}$ specimens initiated on the opposite side of the micro-grooves. The slight decrease of fatigue endurance in Fig. 18 may due to some unbalance of specimen stiffness caused by the longitudinal three grooves.

Figure 19 shows re-plotted data from Fig. 18 as the relationship between $\alpha_{i}$ and $10^{6}$ cycle fatigue strength. The solid actual data includes the influences of work hardening and residual stress. So, they were corrected as follows. The work hardening was measured by micro Vickers hardness, $\mathrm{HV}(0.01)$ at $15 \mu \mathrm{m}$ point from the bottom of microgrooves. The hardness of the base, $t=25 \mu \mathrm{m}, 37.5 \mu \mathrm{m}$, $50 \mu \mathrm{m}$, and $100 \mu \mathrm{m}$ specimens were 191, 226, 238, 249, and 234, respectively. The compressive residual stress at the basilar part was estimated as a half of the strain hardened yield strength of each specimen. ${ }^{25}$ ) The fatigue endurance was corrected by the modified Goodman diagram by using work hardened strength and estimated residual stress. As shown in Fig. 19, the fatigue notch factor $\beta$ seems to be determined by only $\alpha_{i}$ independent of $\rho$ in the microscopic

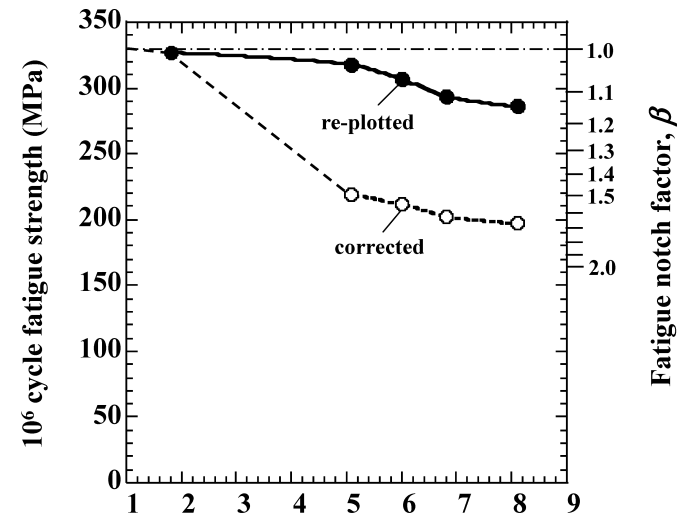

Microscopic stress concentration factor, $\alpha_{\mathrm{i}}$

Fig. 19. Relationship between $\alpha_{i}$ and $10^{6}$ cycle fatigue strength re-plotted from Fig. 18.

stress concentration field. It may be due to the sharp decrease of stress concentration compared to the grain size as shown in Fig. 17. This fact is consistent with the tendency that the dependency of $\beta$ on $\rho$ decreases as the $1 / \rho$ becomes larger, reported by Nisitani. ${ }^{24)}$

For more precise quantitative prediction for the fatigue endurance with microscopic stress concentration, the microscopic non-uniformity of stress distribution caused by anisotropy of each grain orientation and existence of slip band ${ }^{26)}$ must be taking into account in addition to the aforementioned stress relaxation caused by the plastic deformation. The microscopic stress distribution must be separately appraised for the crack initiation and the propagation ${ }^{27)}$ in future studies.

\section{Conclusions}

The effects of microscopic surface stress concentrations on the fatigue endurance of thin-walled high strength steel were estimated by numerical analysis and plane-bending fatigue tests with Schenck-type specimens. The results and conclusions are summarized as follows:

(1) The $\alpha_{i}$ of the $\rho=6 \mu \mathrm{m}$ and $t=50 \mu \mathrm{m}$ microscopic surface ridge monotonously increased with increases of $\theta$ from 1 to 7 in bending mode. A fitted curve was developed for the calculated stress concentration, $\alpha_{\theta}$, derived from the superposition of the principal stresses.

(2) In twisting mode, $\alpha_{i}$ varied from about 4 to 7 . The $\theta$ dependency of $\alpha_{i}$ was smaller than that in bending mode. The empirical rule that the specimen collection direction has less effect in twisting mode fatigue was confirmed by the $\alpha_{i}$ value.

(3) The fatigue notch factor $\beta$ slowly increased with increments of $\alpha_{i}$ until it exceeded about 2, unlike the behavior with increases of the macroscopic stress concentration factor $\alpha_{a}$. This marked difference might be due to differences between their respective stress gradients, which was well described by Nisitani and Endo by using a parameter $\rho$.

(4) The $\theta=0^{\circ}$ micro-groove had little effect on the fatigue endurance in bending. On the other hand, the fatigue cracks of all the $\theta=90^{\circ}$ specimens initiated at the basilar part of the micro-groove without any nonpropagating 
cracks. The fatigue notch factor $\beta$ seems to be determined by only $\alpha_{i}$ independent of $\rho$ in the microscopic stress concentration field.

\section{REFERENCES}

1) K. Tomita, T. Shiozaki, T. Urabe and K. Osawa: Tetsu-to-Hagané, 87 (2001), 557.

2) H. Nisitani and R. Imai: Trans. Jpn. Soc. Mech. Eng. A, 49 (1983), 693.

3) JSME Data Book: Fatigue of Metals (II), 2nd ed., Tokyo, (1984), 26.

4) H. Nisitani, R. Imai and K. Okuzono: Trans. Jpn. Soc. Mech. Eng. A, 53 (1987), 1888.

5) P. Greenfield and D. H. Allen: Gec J. Res., 5 (1987), 129.

6) Y. Murakami, K. Takahashi and T. Yamashita: Trans. Jpn. Soc. Mech. Eng. A, 64 (1998), 1463.

7) T. Takase, K. Setoguchi and T. Wakahara: Trans. Jpn. Soc. Mech. Eng. A, 63 (1997), 1612.

8) R. A. Everett, J. C. Newman and E. P. Phillips: J. Am. Hel. Soc., (2002), 151.

9) M. Kinefuchi: Proc. of JSME/MMD, Toyama, (2003), 755.

10) D. Arola and C. L. Williams: Int. J. Fatigue, 24 (2002), 923.

11) Y. Aono, H. Noguchi, H. Hidaka and T. Hattori: Trans. Jpn. Soc. Mech. Eng. A, 69 (2003), 1186.

12) S. Tamura, T. Mori and N. Hayashi: Proc. Jpn. Soc. Civ. Eng., (2007), 841 .
13) N. E. Frost and D. S. Dugdale: J. Mech. Phys. Solid, 5 (1957), 182.

14) H. Neuber: Kerbspannungslehre, Springer Verlag, Berlin, (1958), 159.

15) G. Sines and J. L. Weisman: Metal Fatigue, McGraw-Hill, New York, (1959), 298.

16) T. Ishibashi: Prevention of Fatigue and Fracture of Metals, Yokendo, Tokyo, (1967), 53

17) S. Koe, H. Nakamura and T. Tsunenari: Zairyo, 35 (1985), 388.

18) Y. Akiniwa: JSMS Fatigue seminar text "Fatigue strength of stress concentrated and defect portion", (2000), 16.

19) M. Nishida: Stress Concentration, enlarged ed. Morikita-shuppan, Tokyo, (2001), 132.

20) M. Kinefuchi and E. Tamura: Proc. of JSME 1, Tokyo, (2003), 119.

21) M. Wakita, T. Kuno, A. Amano, A. Nemoto, K. Saruki and K. Tanaka: J. Hpn. Soc. Spring Eng., 52 (2007), 1.

22) M. Wakita, T. Kuno, A. Amano, A. Nemoto, K. Saruki and K. Tanaka: J. Soc. Mater. Sci. Jpn., 56 (2007), 1096.

23) M. Kinoshita, H. Kimura, K. Osawa, T. Okita, H. Owada and M. Hori: NKK Tec. Rev., No. 145, (1994), 1.

24) H. Nisitani and M. Endo: Trans. Jpn. Soc. Mech. Eng. A, 51 (1985), 784.

25) S.-B. Lee: KSME Journal, 6 (1992), 132.

26) A. Brückner-Foit and X. Huang: Int. J. Fatigue, 28 (2006), 963.

27) S. Toyoda, Y. Ishiguro, Y. Kawabata, K. Sakata, A. Sato and J. Sakai: J. Sol. Mech. Mater. Eng., 3 (2009), 114. 